\title{
Channel Selection Algorithm Optimized for Improved Performance in Cognitive Radio Networks
}

\author{
Joseph Tlouyamma ${ }^{1} \cdot$ Mthulisi Velempini $^{1}$ (D)
}

Accepted: 15 March 2021 / Published online: 26 April 2021

(c) The Author(s) 2021

\begin{abstract}
A major concern in the recent past was the traditional static spectrum allocation which gave rise to spectrum underutilization and scarcity in wireless networks. In an attempt to solve this challenge, cognitive radios technology was proposed. It allows a spectrum to be accessed dynamically by Cognitive radio users or secondary users (SU). Dynamic access can efficiently be achieved by making necessary adjustment to some Medium access control (MAC) layer functionalities such as sensing and channel allocation. MAC protocols play a central role in scheduling sensing periods and channel allocation which ensure that the interference is reduced to a tolerable level. In order to improve the accuracy of sensing algorithm, necessary adjustments should be made at MAC layer. Sensing delays and errors are major challenges in the design of a more accurate spectrum sensing algorithm. This study focuses on designing a channel selection algorithm to efficiently utilize the spectrum. Channels are ordered and grouped to allow faster discovery of channel access opportunities. The ordering is based on descending order of channel's idling probabilities. Grouping of channels ensured that channels are sensed simultaneously. These two techniques greatly reduce delays and maximized throughput of SU. Hence, Extended Generalized Predictive Channel Selection Algorithm, a proposed scheme has significantly performed better than its counterpart (Generalized Predictive Channel Selection Algorithm). Matlab simulation tool was used to simulate and plot the results of the proposed channel selection algorithm.
\end{abstract}

Keywords Cognitive radio $\cdot$ Spectrum sensing $\cdot$ Channel selection algorithm $\cdot$ Medium access control

\section{Abbreviations}

$\begin{array}{ll}\text { SU } & \text { Secondary users } \\ \text { PU } & \text { Primary users } \\ \text { MAC } & \text { Medium access control } \\ \text { GSM } & \text { Global system for mobile communications } \\ \text { WLAN } & \text { Wireless local area network }\end{array}$

Mthulisi Velempini

mthulisi.velempini@ul.ac.za

Joseph Tlouyamma

joseph.tlouyamma@ul.ac.za

1 Department of Computer Science, University of Limpopo, Polokwane, South Africa 


$\begin{array}{ll}\text { TV } & \text { Television } \\ \text { LTE } & \text { Long-term evolution } \\ \text { CR } & \text { Cognitive radio } \\ \text { CRN } & \text { Cognitive radio network } \\ \text { SNR } & \text { Signal-to-noise ratio } \\ \text { EXGPCSA } & \text { Extended generalized predictive channel selection algorithm } \\ \text { CTS } & \text { Clear-to-send } \\ \text { RTS } & \text { Request-to-send } \\ \text { CCC } & \text { Common control channel } \\ \text { ACK } & \text { Acknowledgement } \\ \text { PDF } & \text { Probability density functions } \\ \text { IDT } & \text { Integrated development tools } \\ \text { CSA } & \text { Channel selection algorithm }\end{array}$

\section{Introduction}

Different parts of the spectrum have been traditionally defined to be used by different wireless technology (GSM, WLAN, TV, Military, emergency services or LTE). Unfortunately, due to an increased usage of mobile communication devices, some spectrum bands are crowded while others are hardly used because of fixed spectrum allocation. This leads to spectrum inefficiency challenge depicted in Fig. 1. A primary traffic from the diagram shows the actual data transmission by primary users (PU). Larger parts of the spectrum remain used and these are known as the spectrum holes. Spectrum holes are formed as a result of the under-utilization of finite spectrum.

In order to solve this challenge, cognitive radio (CR) technology is used to efficiently exploit the spectrum white spaces or holes. CR is a radio that is capable of learning the environment in which it operates and change its transmission parameters based on the status (channel occupancy information) of the spectrum band. Regulatory authorities assign certain portion of the spectrum to licensed users or primary users (PU) for data transmission. A diagram in Fig. 2 shows a dynamic network environment where Secondary users

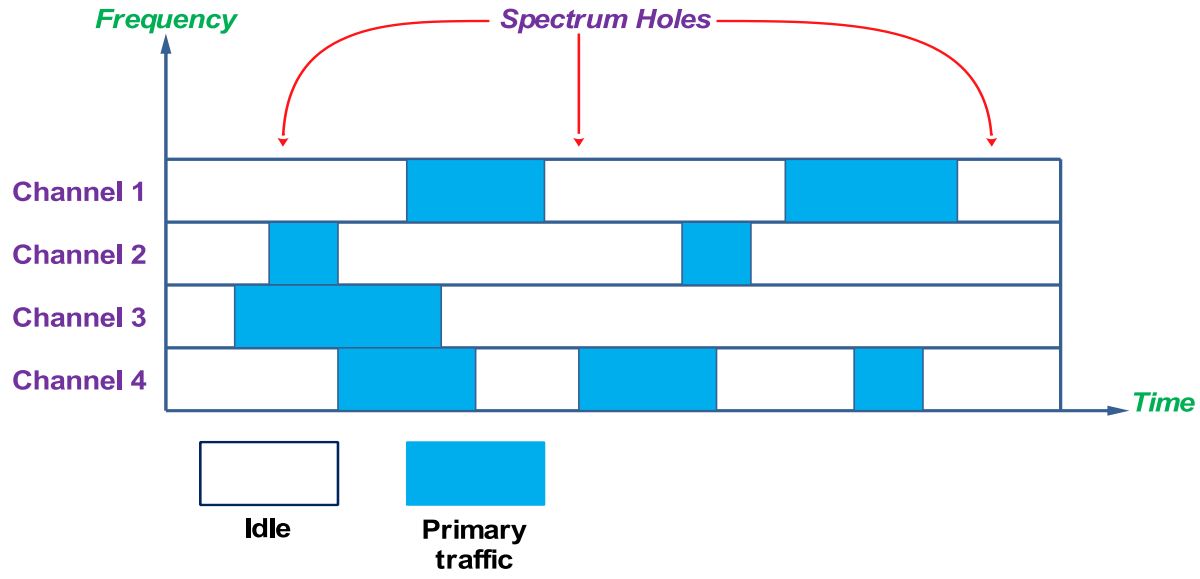

Fig. 1 Fixed spectrum allocation in TV white spaces 

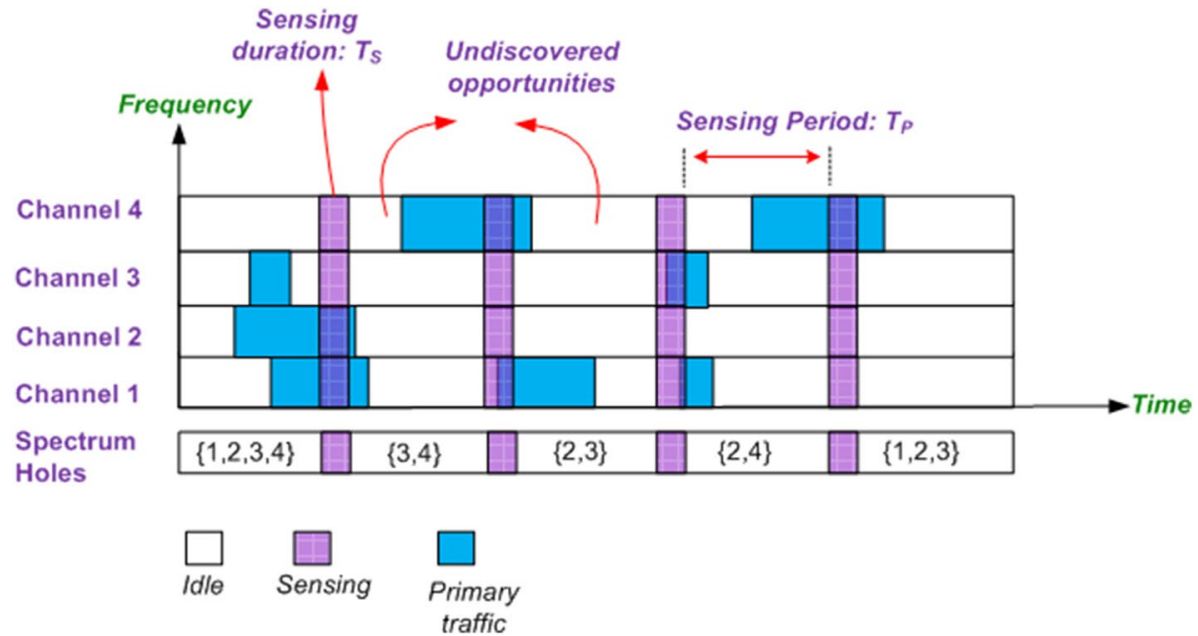

Fig. 2 Dynamic spectrum allocation in TV white spaces [1]

(SU) opportunistically transmit their data in the channels assigned to PUs. Data transmission has to be done after sensing to avoid interference with PUs.

There are two approaches to spectrum sensing in CR networks. That is, sensing at physical layer and MAC layer. Sensing a spectrum at the physical layer makes adaptation of modulation schemes and parameters for detecting and measuring the signals transmitted by PUs from different channel while MAC layer makes a decision as when SUs have to sense which channels [2]. MAC layer is responsible for searching of channels, transmissions, and scheduling time slots for sensing. MAC has received far less attention as compared to the physical layer sensing. Spectrum sensing function belongs to physical layer but the coordination and scheduling of SUs is the responsibility of MAC layer.

Proper coordination and scheduling of SUs lead to better utilization of spectrum resources. A key technology in efficient use of the spectrum is spectrum sensing. Sensing must be performed accurately to avoid interference and degradation of performance. Secondly, a seamless communication must be maintained during spectrum mobility. If a PU is detected in the current band a SU is transmitting in, SU will have to move to a new band to continue with its data transmission. This movement is made possible by spectrum mobility function. A perfect timing has to be performed during spectrum handoff. Spectrum handoff happens when SU ceases its transmission from one spectrum and migrates to the other.

The major causes of delays in data transmission in cognitive radio networks are cooperative sensing and sharing of the sensed results. In this study, these challenges are addressed in order to maximize the throughput of SU. Hence, we proposed a channel selection algorithm to intelligently select PU channels that are free and ready to be used opportunistically by SUs for data transmission. The channels with the highest probability of being free from PUs are selected for sensing. Delays incurred during cooperative sensing are dealt with through sensing multiple channels at the same time (parallel sensing) and the use of probabilistic models to model delays in sensing and sharing of spectral observations.

In order for SUs to reliably detect the presence of PUs in the channel and reduce sensing delays, we incorporate cooperative spectrum sensing and set a rule which ensures that only cooperating SUs with higher signal-to-noise ratio (SNR) share their sensed results. For 
this, we proposed an algorithm called extended generalized predictive channel selection algorithm (EXGPCSA). This algorithm is an improved version of an existing algorithm called generalized predictive channel selection algorithm [3].

\section{Related Work}

To improve the performance of spectrum sensing algorithms and reliability of sensing results, most studies proposed cooperative spectrum sensing where SUs collaborate in finding channels [4-9]. The main objective of these studies is to address constraints which degrade performance of sensing algorithms such as multi-hidden terminal problem, multipath fading, and receiver uncertainty and shadowing. Cooperative spectrum sensing algorithms are used to efficiently exploit the spatial diversity of SUs.

Authors in [10] proposed sequential algorithm for maximization of achievable throughput of SUs in multi-channel CR network. The time it takes SUs to sense the spectrum has been considered using effective rate criterion where trade-offs between sensing time and sensing accuracy are captured. Constrained dynamic program is formulated in this study for optimal sensing duration scheme. This policy determines when to stop sensing and ensures optimal channel access to choose a set of available channels for data transmission. To improve reliability and accuracy, cooperative sensing is employed in [11] where cooperative overheads are reduced by improving the accuracy of local spectrum sensing. The study proposed two-stage local spectrum sensing approach. In the first stage, each SU measures its local environment for the availability of channels and in the second stage; the results are combined using fuzzy logic in order to detect the presence of PU in the spectrum.

To achieve greater throughput, a group of SUs must cooperate in sensing multiple channels within one sensing time slot or period [12]. This is achieved through parallel sensing technique. Reliable techniques of combining of sensing results and selection of cooperating SUs are studied in [12]. The trade-off between accuracy and efficiency in cooperative spectrum sensing has been addressed through independently selecting heterogeneous SUs for parallel sensing such that different groups of SUs can sense multiple channels simultaneously. The main design objective of the study in [13] was to limit the interference of PUs while ensuring maximum throughput of SUs. Three components have been integrated in the joint design: spectrum sensor, access strategy, and sensing strategy. Spectrum sensor ensures that more spectrum opportunities are identified. Authors used receiver operating characteristics of spectrum sensor to capture the trade-off between miss detection and false alarms.

A study in [14] proposes a fusion scheme and an optimization of cooperative sensing by choosing a value of $K$ that maximizes SU's throughput. Authors used K-out-of-N fusion technique as basis for the formulation of optimization problem and a parameter $\mathrm{k}$ was chosen as optimization variable. To obtain optimal sensing duration and value of $\mathrm{k}$, an iterative algorithm was proposed. Proposed in [15] is the majority or half voting rule in which a channel is unavailable if $\mathrm{N}$ out of $\mathrm{K}$ SUs votes that indeed a channel is occupied by PU where $\mathrm{N}>(\mathrm{K} / 2)$. In special case where $\mathrm{N}=\mathrm{K}$ and $\mathrm{N}=1$, AND rule and OR rule is applied. The assumption made from this study was that all SUs participating in decision making must have reached a set threshold. This requirement in reality is not ease to achieve. Channel selection, multicast routing, call admission control and scheduling were considered in [16] to appropriately use network resources. The proposed cross-layer scheme did not 
only reduce multicast period but also efficiently addressed the blocking problem. Authors considered multi-radio technology but did not clearly show how self-interference could be avoided.

The objective of the study in [17] was to increase throughput and reduced channel switching rate. The study considered opportunistic IEEE 802.11 MAC to intelligently tackle problems in channel switching and the receiver synchronization with the sender to allow seamless communication. To reduce channel switching, channel selection scheme based on successful data frame transmission was proposed. In another study in [18], authors used Markov decision process to model the channel selection problem. Real-time reinforcement learning algorithm enhanced learning through cooperative sensing. The algorithm achieved higher packet success rate compared to conventional channel selection algorithm without a learning mechanism. The attainability of the required channel and suitability of bandwidth were considered in [19] as factors that play crucial role in channel scheduling. The study proposed an algorithm to allocate optimal idle channel, optimal multiple idle channels and optimal multiple channels [20]. The study reported low transmission loss and high throughput compared to other contemporary methods.

Joint channel assignment and adaptive multi-input-multi-output (MIMO) enhances the performance of the system by selecting MIMO operating mode for every idling channels according to the surrounding radio frequency environment [21]. It also achieves greater performance by selecting channel with the highest throughput. The study has shown significant performance improvement in achievable throughput under various traffic and network conditions. A technique called Navien Bayes classifier was proposed in [22] to discover spectrum opportunities. The use of historic data enabled SU to choose the channel with the lowest probability of a channel being busy for data transmission. The study further considered time series prediction to predict the likelihood of channels being busy in near future.

Authors in [23] suggested that the use of stochastic approach under the hybrid with Invasive weed optimization algorithm increases spectrum hand off efficiency in CR networks. The channel selection accuracy of this approach was found to be $97.8 \%$ effective than other conventional methods. The support vector machine and pre-emptive resume priority queueing model $\mathrm{M} / \mathrm{M} / 1$ enhanced the performance of CR systems by selecting best possible network channel [24]. The study reported $68.7 \%$ of SU throughput and a decrease in the number of spectrum handoffs. The training accuracy of the support vector machine method increased to $97.6 \%$ outperforming other conventional methods. Token passing scheme designed to switch channels between SU and PU was considered in study [25] to achieve maximum spectrum efficiency and minimize energy consumption. It was concluded that token passing took lesser time in switching the channels and that it positively influenced the throughput and saved energy consumption. In their study in [26], authors designed an algorithm with capabilities of adapting its rate and opportunistically make use of spectrum White Spaces when the channels conditions are not known and when the channel state information is not available. Numeric results demonstrated that the algorithm significantly achieved better performance compared to some of the state-of-the-art schemes. 


\section{System Model and Algorithms}

A scientific way of solving complex challenges is through models and algorithms which ensure better understanding of a problem at hand and the derivation of better solutions. Hence, presented in this section are models, algorithms, and parameters necessary to design and evaluate the efficiency of EXGPCSA.

\subsection{Receiver/Transmitter Operation}

We consider a group of SUs communicating in a simple multi-hop CRN. Each SU is equipped with a single radio for both sensing and transmission. Unfortunately, sensing and data transmission cannot be performed simultaneously. All SUs stop transmitting when sensing the spectrum. This is one of the requirements in the 802.22 IEEE standards which necessary to give SU time to detect the presence of PU signal.

To manage properly the coordination of SUs, we use the listen before talk strategy in which a transmitter listens to the medium or wireless channel before transmitting data packet. To avoid interference amongst transmitters (SUs), clear-to-send (CTS) and request-to-send (RTS) control packets are flooded to the network. RTS is meant to check if there is any other transmitter using wireless channel. If a channel is currently being occupied, transmitter backs off for a random amount of time before trying again. CTS data packet is used to alert the transmitter that there is a free data channel between the current transmitter and the receiver.

The transmitter-receiver pair start data exchange after CTS has been received by the transmitter. Once a data packet has successfully been received at the destination, the receiving SU will acknowledge the receipt of data packet by sending an ACK packet. Transmitting SU will have to transmit the same data packet if no ACK packet has been received. Common control channel (CCC) is used for exchange of control packets.

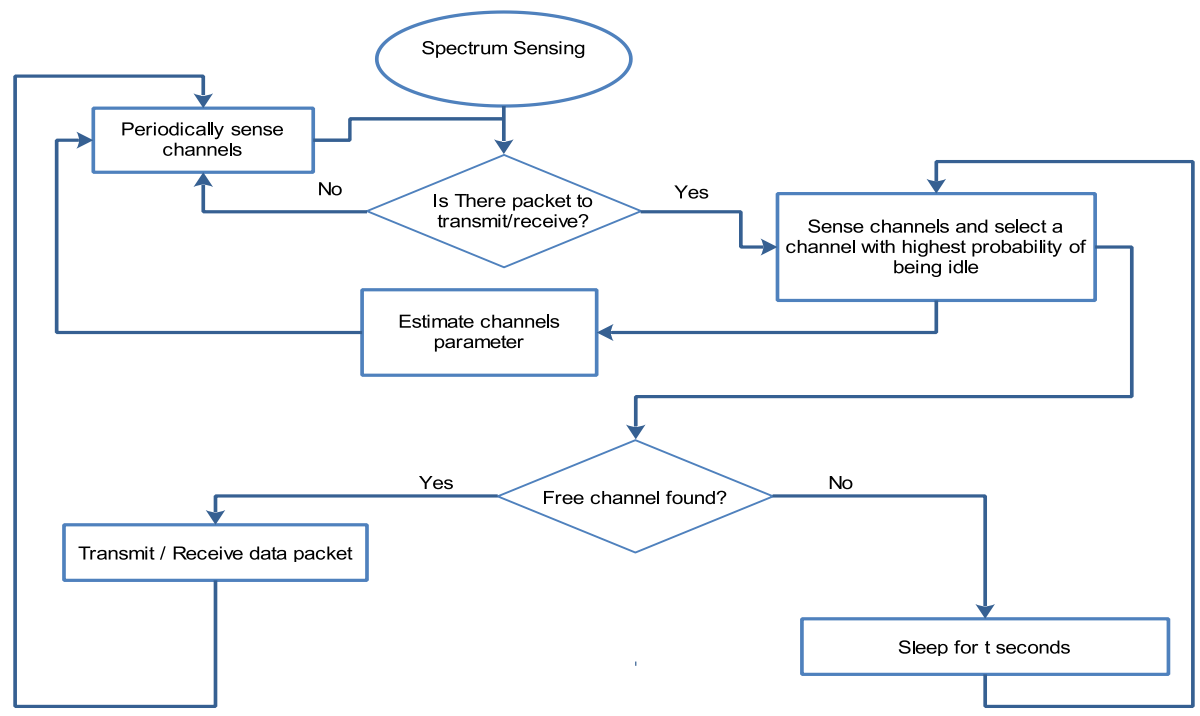

Fig. 3 Sensing strategy flow diagram 
Figure 3 depicts sensing and data transmission by SUs. SUs periodically senses the spectrum and estimate channel parameters. Data is transmitted only when free channel is available else SU will sleep for a specific amount of time before trying again.

\subsection{Channel Model}

We consider $\mathrm{N}$ channels being opportunistically accessed by SUs where each channel is licensed to PU. The ON times (SU's channel usage time)/OFF times (PU's inter-arrival time) on a channel $\mathrm{c}$ are modelled using probability density functions (PDF) represented by $f_{X}{ }^{c}$ and $f_{Y}{ }^{c}$ respectively. The assumption is that the distributions of ON/OFF time are independent and not the same. This is validated in [27].

As shown in Fig. 3, SUs are required to search for a channel starting at $t_{s}$ for $t$ duration. The lengths of $\mathrm{ON}$ and $\mathrm{OFF}$ periods are represented by $\mathrm{X}^{\mathrm{c}}$ and $\mathrm{Y}^{\mathrm{c}}$ respectively. If we assume that these periods are exponentially distributed, then

$$
\mathrm{f}_{\mathrm{x}}^{\mathrm{c}}(x)=\mu_{x^{c}} e^{-\mu} x^{c x}, \quad \forall \mu_{x^{c}}=\frac{1}{E X^{c}(x)}
$$

and

$$
\mathrm{f}_{\mathrm{Y}}^{\mathrm{c}}(y)=\mu_{y^{c}} e^{-\mu} y^{c y}, \quad \forall \mu_{y^{c}}=\frac{1}{E Y^{\mathrm{c}}(y)}
$$

where $\mu$ is a rate parameter and $\mu \geq 0, E X^{c}(x)$ and $E Y^{\mathrm{c}}(y)$ are mean values of the distribution, $\mathrm{f}_{\mathrm{x}}^{\mathrm{c}}(x)$ is the PDF of the ON periods on channel $\mathrm{c}$ and $\mathrm{f}_{\mathrm{Y}}{ }^{\mathrm{c}}(y)$ is the PDF of the OFF periods on channel $\mathrm{c}$.

To find Mean values of ON periods, we use Eq. 3,

$$
\begin{aligned}
E X^{c}(x) & =\int_{0}^{\infty} \operatorname{fxc}(x) d x \\
& =\int_{0}^{\infty} \mu_{x^{c}} e^{-\mu} x^{c x} d x
\end{aligned}
$$

To evaluate the integral using integration by parts, we then havesimilarly for OFF periods

$$
\begin{gathered}
E X^{c}(x)=\frac{1}{\mu} \\
E Y^{c}(y)=\int_{0}^{\infty} \mathrm{fYc}(y) d y \\
=\int_{0}^{\infty} \mu_{y^{c}} e^{-\mu y^{c y} d y}
\end{gathered}
$$

Applying integration by parts we get, 


$$
E Y^{c}(y)=\frac{1}{\mu}
$$

\subsection{Proposed Channel Selection Algorithm}

There are two classifications of channel selection algorithms of MAC protocols in multichannel cognitive radio network; (1i) ON/OFF distributions based channel selection algorithm where channel statistics are known in advance or can be estimated at the run time. These types of algorithms rely heavily on the first and second order statistics of ON/OFF time distributions and it's often very difficult to predict the distributions of ON/OFF times at the run time and (2ii) learning-based channel selection algorithm in which channel usage pattern is not known to SUs but they can be learnt from usage patterns or parameters.

The learning based algorithms perform better in terms of estimating channel usage pattern and finding idle channels with minimal delay [27]. For this reason, our study focuses on modifying a channel selection algorithm which is based on ON/OFF time distributions studied in [28].

In this study, we address two key issues; (i) we determine how SUs can efficiently and effectively select channels for sensing. From Fig. 4 there are N SUs sensing N channels. SUs should sequentially sense all channels until an idle channel is found. In this case a question may be; how long, on average does it take SUs to find an idling channel. If SUs can intelligently locate channels with the highest probability of being idle, then sensing delays can be greatly minimized. We achieve this by implementing channel selection algorithm to intelligently select free PU channels.

In (ii) we optimize $\mathrm{T}_{\mathrm{p}}{ }^{\mathrm{c}}$, which is the sensing period on channel c such that less transmission opportunities are missed. The shorter the $\mathrm{T}_{\mathrm{p}}{ }^{\mathrm{c}}$, the more the overheads are incurred, and

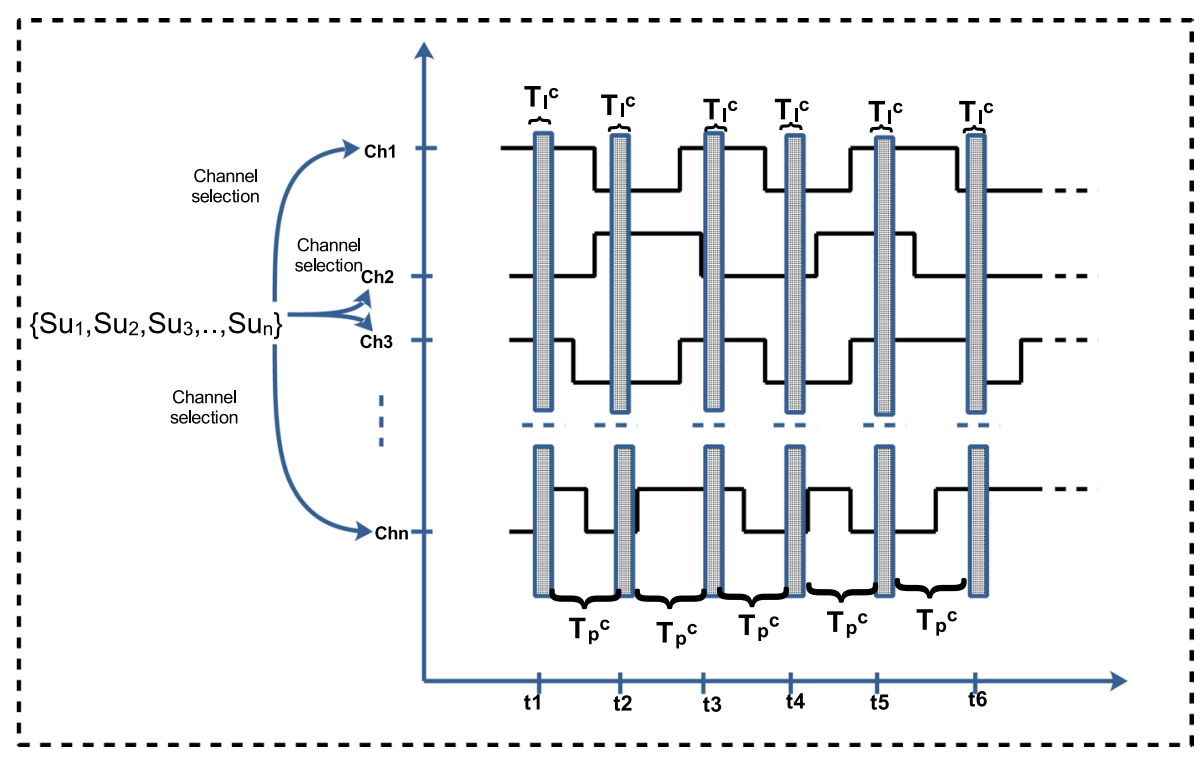

Fig. 4 graphical representation of SU channel selection 
this ultimately leads to compromised throughput while on the other hand blindly increasing $\mathrm{T}_{\mathrm{p}}{ }^{\mathrm{c}}$ leads to more opportunities being missed. We don't focus on $\mathrm{T}_{\mathrm{I}}^{\mathrm{c}}$ (sensing interval on channel c) since it is mainly determined by the physical layer as per 802.22 standards. We therefore make few assumptions for most of physical layer parameters.

Shown in Fig. 4 is how SUs sense and select channels. $T_{I}^{c}$ is the sensing time or interval and $\mathrm{T}_{\mathrm{P}}{ }^{\mathrm{c}}$ is the sensing period. It is very critical to consider how long and how often to sense the spectrum. Hence this was considered during the design of our algorithm. An algorithm for sensing multiple channels at the same time was designed. This algorithm is explained and presented in detail in the sequel.

We have taken a probabilistic approach to predict the state of a channel at a particular point in time. To efficiently predict using probabilities, we allow SUs to maintain a believe vector, $\boldsymbol{V}_{p(\boldsymbol{c})}(t)$ which has $\mathrm{N}$ dimensions in two sets or groups and if we let $\boldsymbol{O}_{p(\boldsymbol{c})}$ and $\boldsymbol{q}_{p(\boldsymbol{c})}$ be the probabilities that channels are free from PU, then a believe vector containing this probabilities in descending order is defined as follows:

$$
\boldsymbol{V}_{p(\boldsymbol{c})}(\boldsymbol{t})=\left\{\left\{\boldsymbol{O}_{p(\mathbf{1})}, \boldsymbol{O}_{p(\mathbf{2})}, \boldsymbol{O}_{p(\mathbf{3})}, \ldots, \boldsymbol{O}_{p(N)}\right\},\left\{\boldsymbol{q}_{p(\mathbf{1})}, \boldsymbol{q}_{p(\mathbf{2})}, \boldsymbol{q}_{p(\mathbf{3})}, \ldots, \boldsymbol{q}_{p(N)}\right\}\right\}
$$

The elements of $\boldsymbol{V}_{p(c)}(t)$ are arranged such that

$$
\boldsymbol{O}_{p(\mathbf{1})}>\boldsymbol{O}_{p(\mathbf{2})}>\boldsymbol{O}_{p(\mathbf{3})},>\ldots, \boldsymbol{O}_{p(N)} \text { and } \boldsymbol{q}_{p(\mathbf{1})}>\boldsymbol{q}_{p(\mathbf{2})}>\boldsymbol{q}_{p(\mathbf{3})},>\ldots, \boldsymbol{q}_{p(N)} .
$$

Using this $\boldsymbol{V}_{p(c)}(t)$ (believe vector), SUs selects a channel with the highest probability of being free from each group. This is equivalent to say:

$$
\mathbf{c}^{*}(\boldsymbol{t})=\left\{\arg \left\{\max _{1 \leq c \leq N} \boldsymbol{V}_{p(\boldsymbol{c})}(\boldsymbol{t})\right\}, \arg \left\{\max _{1 \leq \boldsymbol{c} \leq N} \boldsymbol{V}_{q(\boldsymbol{c})}(\boldsymbol{t})\right\}\right\}
$$

If a channel with the highest probability i.e., $\mathrm{c}^{*}$ has been sensed and is occupied, SUs sense the next channel with the highest probability and this is done repeatedly until a free channel is found. An idle channel is then used by SUs for $t$ duration. In order to avoid interference SUs have to sense a channel for $\boldsymbol{t}$ duration as shown in Fig. 3.

\section{Algorithm 1: channel selection Algorithm}

1. Use results from slot $i-1$

2. if $q_{p(c)} \leftarrow p_{1}(i-c)$

3. then

4. Sensing_results $\leftarrow 1$

5. else if $q_{p(c)} \leftarrow p_{0}(i-c)$

6. then

7. sensing results $\leftarrow 0$

8. $\operatorname{usec}^{*}(t)$

9. $\operatorname{select}\left\{\arg \left\{\max _{1 \leq c \leq N} V_{p(c)}(t)\right\}, \arg \left\{\max _{1 \leq c \leq N} V_{q(c)}(t)\right\}\right\}$

10. if channel $c^{*} \leftarrow 0$

11. then

12. SU packet transmission

13. else if channel $c^{*} \leftarrow 1$

14. select another channel with $\left\{\arg \left\{\max _{1 \leq c \leq N} V_{p(c)}(t)\right\}, \arg \left\{\max _{1 \leq c \leq N} V_{q(c)}(t)\right\}\right\}$ 


\section{Simulation Scenarios}

This section contains simulation environment, parameters and models used to generate the results of this study.

Identification of key technologies to be incorporated in the development of any algorithm is very critical. Different Integrated development tools (IDTs) are suitable for different tasks. The rate at which spectral opportunities are discovered partially determines the efficiency of the spectrum sensing algorithm. Many network simulators have been developed to help the researchers to evaluate their algorithms and/or protocols. A best network simulator in the context of spectrum sensing can be determined by its flexibility in employment of custom MAC and physical layer algorithms, support of default communication protocols and the ability to employ custom sensing algorithms. We present and discuss metrics considered in the design of the proposed algorithm.

Figure 5 shows simulation grid where SUs sense and share observations about the spectrum in $1000 \mathrm{~m}^{2}$ area. A simulation was carried for $200 \mathrm{~s}$ with 30 SUs in a network. All SUs in a network are equipped with one radio for sensing and data transmission. SUs communicate with each other through fusion node.

\subsection{Probability of Detection}

An optimization of threshold level has shown to increase the probability of detection while minimizing sensing errors (probability of miss detection and probability of false alarm) [28]. Next we determine the threshold level which serves as a constraint for the detection probability and probability of false alarm. We choose a test statistic $\boldsymbol{T}_{\boldsymbol{L}}$ for energy detection such that false alarm occurs whenever $\boldsymbol{T}_{L}$ is greater than $\boldsymbol{T}_{\boldsymbol{h}}$ where $\boldsymbol{T}_{\boldsymbol{h}}$ is the threshold level.

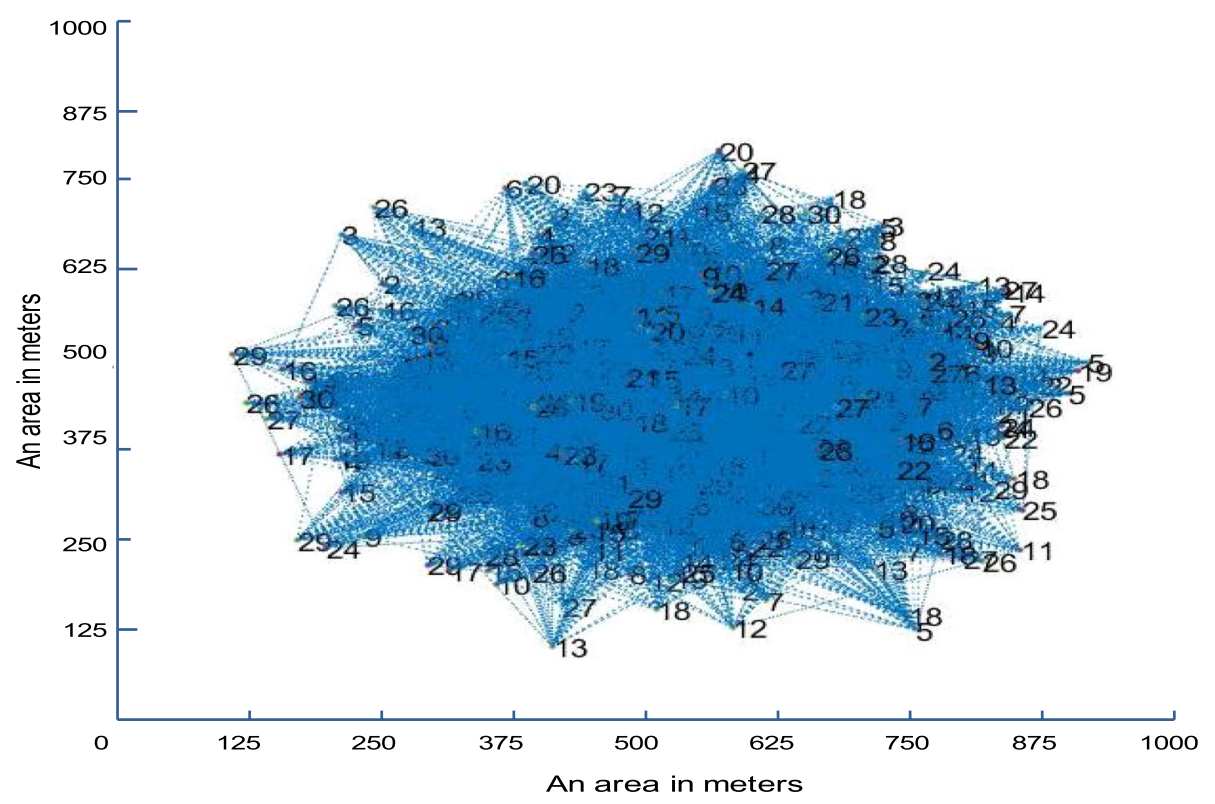

Fig. 5 Collaborative sensing and sharing in cognitive radio network environment 
Both test statistic and threshold values are given by:

$$
T_{L}(y)=\frac{1}{L} \sum_{n=1}^{L}|y(n)|^{2}
$$

where $\boldsymbol{L}$ is the number of samples and $\boldsymbol{y}(\boldsymbol{n})$ is the received signal sample of a SU.

$$
T_{h}(x) \frac{1}{\sqrt{\alpha L}} \int_{x}^{\infty} \exp (x / 2) d x+1, \quad \forall \alpha \epsilon\{1,2,3\}
$$

\subsection{Delay in Finding Idle Channels}

This is given by $D_{c}=D_{s}+W_{t}$ where $D_{c}$ is the delay in finding available channel, $D_{s}$ is the searching duration and $W_{t}$ is the waiting time.

The average delay is then given by

$$
A v_{D}(t)=\frac{1}{n} \int_{0}^{\infty} D_{c}(t) d t
$$

where $\mathrm{n}$ is the number of $\mathrm{SU}$ in a network.

\subsection{Channel Switch Rate}

The rate at which channels are switched. $\boldsymbol{C}_{\boldsymbol{R}}(t)$ is calculated as a fraction of number of channel switches of SU sender to the time period $t$.

\subsection{Throughput}

The throughput of SUs $\boldsymbol{T}(\boldsymbol{t})$ is calculated by taking a ratio of the product of successfully transmitted data frames and the size of frames in bits to the total time $t$ elapsed during simulation. The throughput of SUs is given in [3] by:

$$
T(t)=\frac{F_{N}(t) * \text { Frame size }[\text { bits }]}{t}
$$

where $\boldsymbol{F}_{N}(\mathbf{t})$ is the number of successfully transmitted frames until time $\boldsymbol{t}$. The average throughput of SUs is then given by:

$$
\lim _{n \rightarrow \infty} T(t)=\lim _{n \rightarrow \infty} \frac{F_{N}(t) * \text { Frame size }[\text { bits }]}{t}
$$

Table 1 presents the simulation parameters used to simulate EXGPCSA. These parameters were set for all network scenarios and simulated under a very dynamic environment to mimic the actual cognitive radio network environment. 
Table 1 Simulation parameters

\begin{tabular}{ll}
\hline Parameters & Values \\
\hline Network grid & $1000 \mathrm{~m} \times 1000 \mathrm{~m}$ \\
Network simulator & MATLAB R2015a \\
Simulation time & $200 \mathrm{~s}$ \\
Transmission range & Maximum 1000 m \\
Traffic type & Periodic \\
Number of secondary nodes Ns & 30 \\
Number of radios & 1 \\
Load on the network & $80 \%$ \\
Super frame & $160 \mathrm{~ms}$ \\
Frame size & $10 \mathrm{~ms}$ \\
Modulation scheme & QPSK \\
Maximum transmission power for secondary & $1 \mu \mathrm{W}$ \\
$\quad$ nodes & \\
Collaborative sensing & OR \\
Operating System & Windows 7 OS
\end{tabular}

\section{Results and Discussions}

A Matlab simulation tool was used to simulate a cognitive radio network in $1000 \mathrm{~m} \times 1000 \mathrm{~m}$ grid and a simulation time was set to $200 \mathrm{~s}$. The performance metrics used during the simulation to evaluate the efficiency of EXGPCSA are the probability of detection, delays in finding the available channels for transmission, the rate at which channels are switched as well as throughput.

A level of complexity at which data is aggregated can influence the detection probability. Whenever complex measures are considered, the detection tends to be more accurate but low. As it can be evident from Fig. 6, OR rule requires a simple aggregation technique which allows faster data aggregation and higher detection with lower accuracy. A major drawback of this rule is that, an increase in probability of detection leads to an increase in the probability of detection. In contrary, AND rule decreases the probability of detection with the decrease in probability of false alarm. Although results obtained using this rule are accurate and reliable, the chances of detecting PU signal are very low.

In $\mathrm{N}$-out-M aggregation rule, $\mathrm{N}$ can be chosen in such a way that the probability of detection remains in an acceptable level. In such cases, the detection would be very accurate. The manner in which data is aggregated using AND, and OR rule, introduces delays due to robustness in data aggregation. Although detection of the signal using these two rules is accurate and reliable, they cannot give good results in application where faster data aggregation and data transmission is required.

The ordering and selection of channels influences the transmission rate of data. EXGPCSA allows multiple channels to be sensed simultaneously while ordering them in the descending order of their idling probabilities. This greatly reduces delays in available channels for data transmission as it can be shown in Fig. 7. As it can be noticed, delays in finding the available channels increases with the increase in inter-sensing interval. If a channel is being sensed for longer periods, delays increase which compromises the throughput.

Figure 8 shows the rate at which nodes switch between channels when inter-sensing intervals are increased. SUs switch from one channel to the other searching for free 


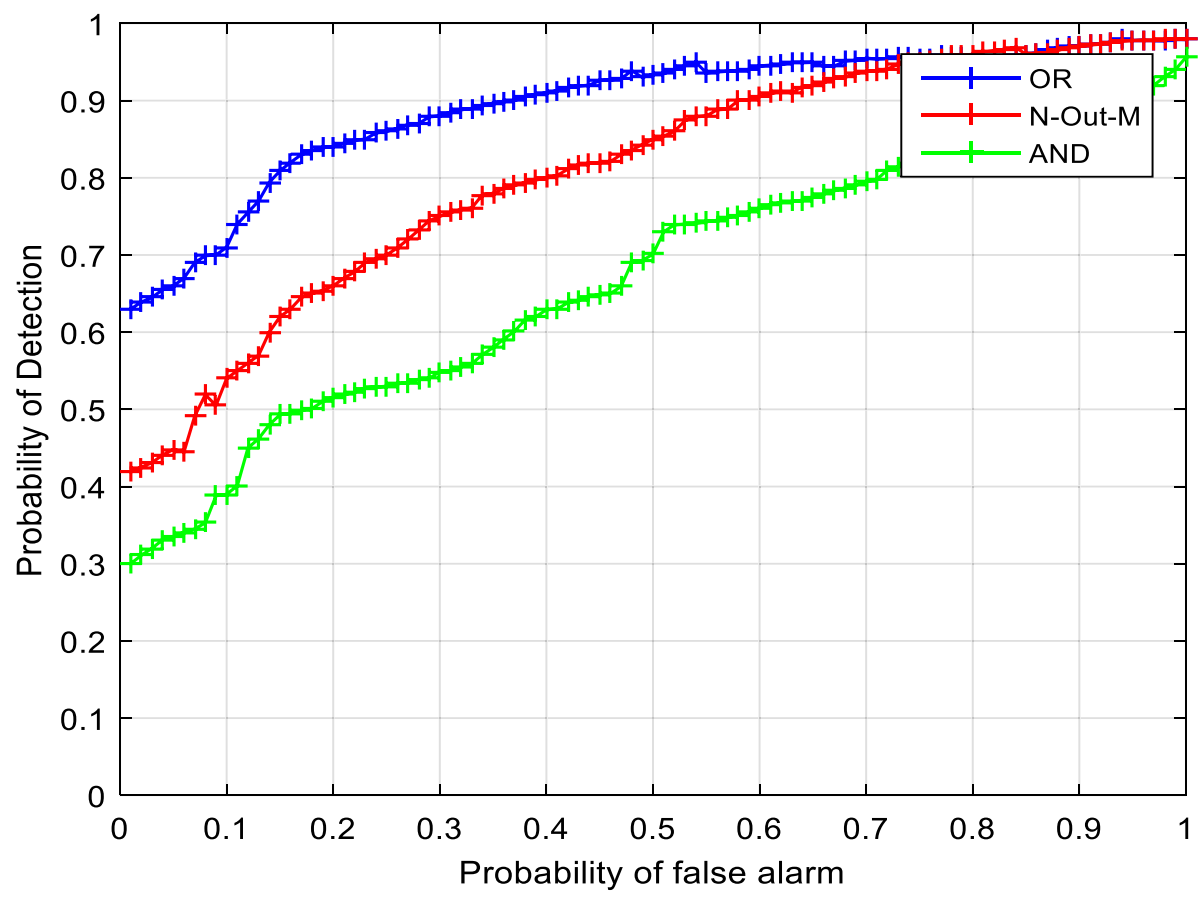

Fig. 6 Comparison of collaboration rules for better detection probability

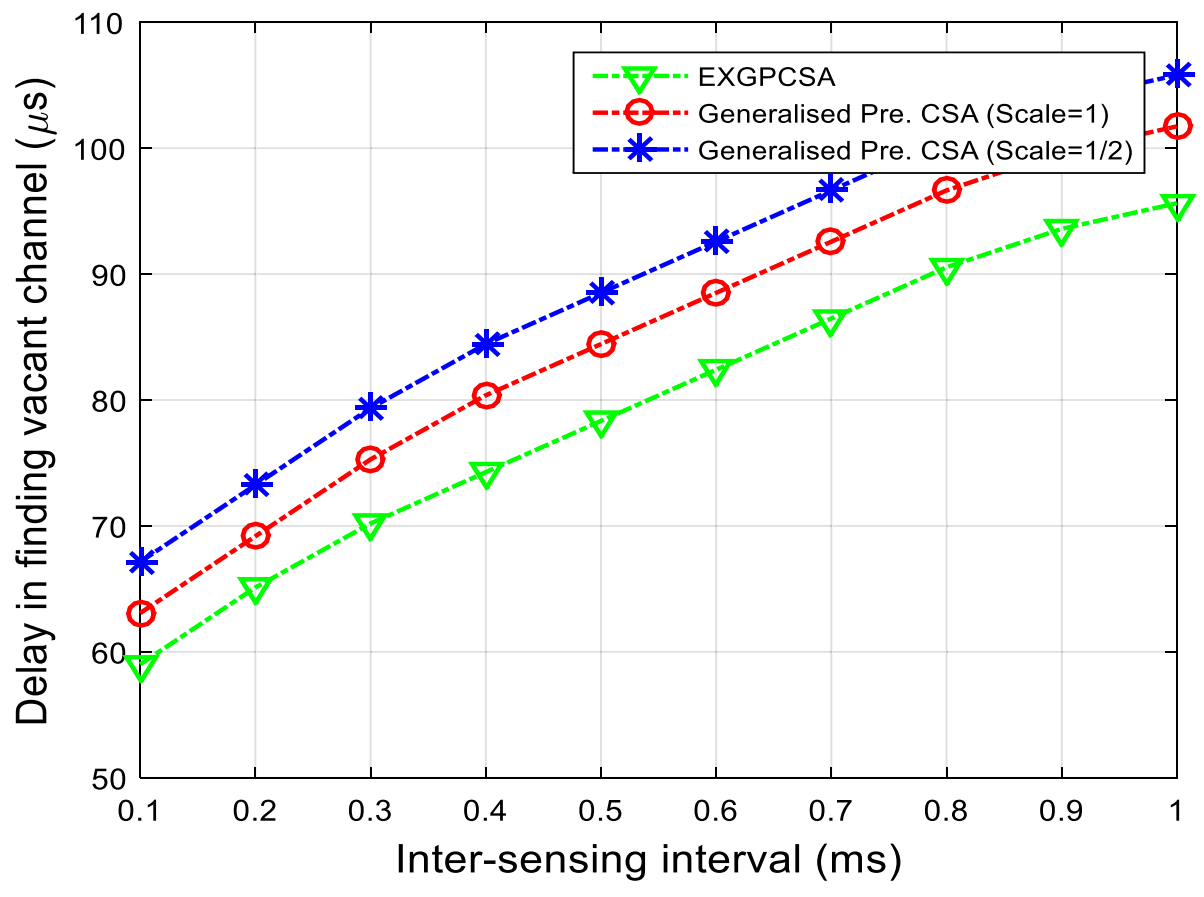

Fig. 7 Delay in finding idle channels over 6 channels in cognitive radio networks 


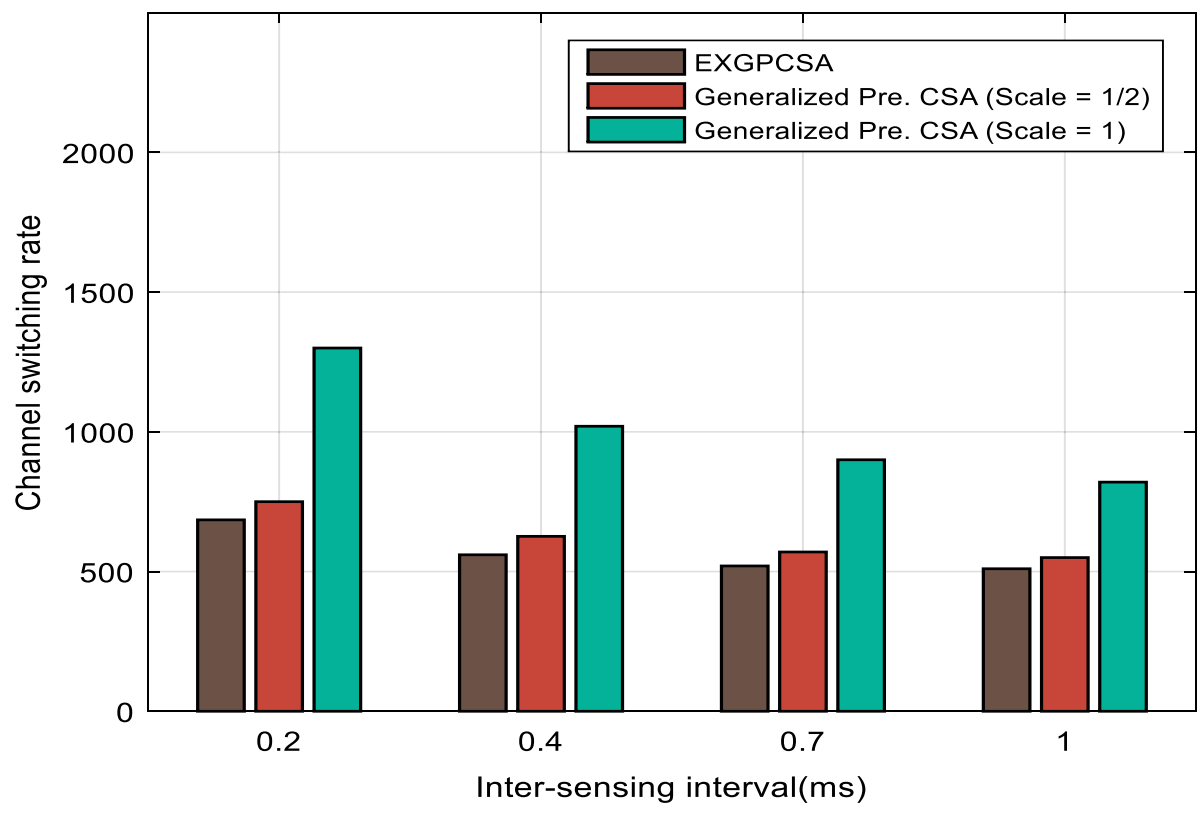

Fig. 8 The rate at which channels are switched in a cognitive radio network

channels for transmission. It is desirable to have few channel searches before finding the next available channel to reduce searching delays. Channel switching rate remains higher when inter-sensing interval is small. This is due to the fact that sensing is performed within a very short period of time and this causes high overheads and more channel switches. As a result, it is difficult to certainly detect a signal, hence more channels switches. Knowing the channels that are likely to be idling reduces channel switches and searching delays. Hence EXGPCSA uses channel selection algorithm which intelligently selects channels to be sensed. This greatly reduces the number of channel switches since only channels with the highest probabilities of being free are selected for sensing. It can be observed from Fig. 8 that channel switching decreases with the increase in inter-sensing interval. Whenever sensing for extended period of time, the sensing results are obtained with certainty and more likely to be accurate. What is crucial then would be to select those channels that are idling. If only idling channels are selected, then there would be no need to switch between multiple channels, hence less channel switching occurrences.

Sensing duration directly has an impact on the network throughput. A channel that has been sensed for a longer duration delays data transmission since data can only be transmitted after sensing is completed. This is depicted in Fig. 9. This figure shows the throughput ranges of SUs in a CR network for different inter-sensing intervals.

The throughput generated by SUs when EXGPCSA was simulated for $2 \mathrm{~ms}$ is in the range of 25.5-30.5 kbps while the throughput of the Generalises Pre CSA was in the range of 24-29.5 kbps. Considering average throughput from these ranges, we observed that EXGPCSA has higher throughput when inter-sensing interval is $2 \mathrm{~ms}$. The same trend was observed for $6,10,14$ and $18 \mathrm{~ms}$.

It can also be observed that EXGPCSA outperformed generalized predictive CSA for $2 \mathrm{~ms}$ scenario. The results show that $25 \%$ of SUs in EXGPCSA recorded achievable throughput which is above $29 \mathrm{kbps}$. It was in this sensing interval scenario that all the 


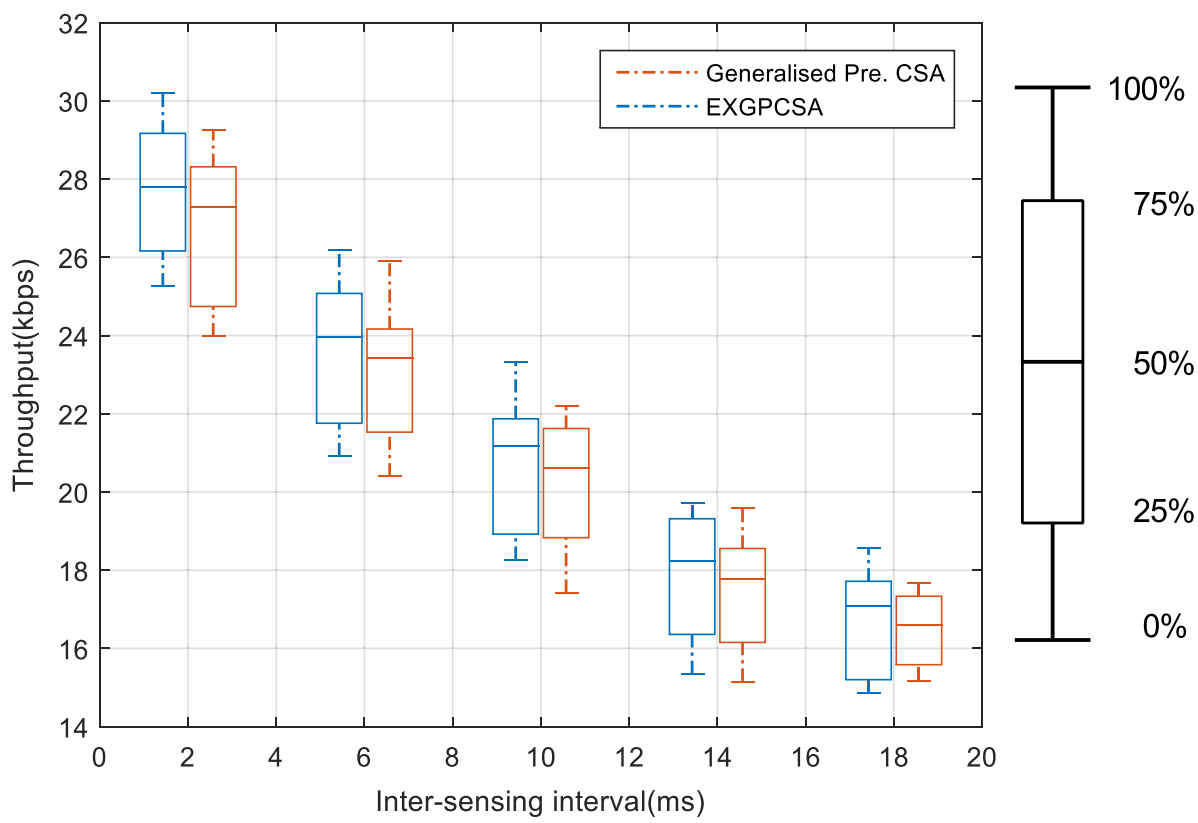

Fig. 9 Box and whisker plot representing individual throughput of SUs in a cognitive radio network

schemes had the highest achievable throughput. The throughputs generally dropped as the inter-sensing intervals were increased. There may be many causes of this but one of them is delay in sensing. Increasing sensing interval causes delays in data transmission and in many cases may lead to spectrum under-utilization due missed opportunities. These factors have degraded achievable throughput as depicted in Fig. 9.

\section{Conclusions}

A robust approach was taken in designing and implementing EXGPCSA in which the collaborative sensing, parallel sensing, and data aggregation rules were considered. This approach is very complex in nature and required necessary techniques to achieve better performance. Hence, a channel selection technique and sensing strategies were employed to intelligently enable SUs to adapt to their environment. EXGPCSA consistently achieved better results compared to the generalized predictive CSA. We can therefore, conclude that EXGPCSA significantly outperformed the generalized predictive CSA.

Funding This work is based on the research supported in part by the National Research Foundation" of South Africa for the Grant, Unique Grant No. 94077" and the Centre for Scientific Industrial and Research (CSIR).

Data Availability Data can be shared and is available on request. Data can be requested by sending an email to the main author. 


\section{Declarations}

Conflict of interest The authors declare that they have no conflict of interest.

Open Access This article is licensed under a Creative Commons Attribution 4.0 International License, which permits use, sharing, adaptation, distribution and reproduction in any medium or format, as long as you give appropriate credit to the original author(s) and the source, provide a link to the Creative Commons licence, and indicate if changes were made. The images or other third party material in this article are included in the article's Creative Commons licence, unless indicated otherwise in a credit line to the material. If material is not included in the article's Creative Commons licence and your intended use is not permitted by statutory regulation or exceeds the permitted use, you will need to obtain permission directly from the copyright holder. To view a copy of this licence, visit http://creativecommons.org/licenses/by/4.0/.

\section{References}

1. Bayhan, S., \& Alagöz, F. (2017) MAC layer spectrum sensing analysis of a logical channel cognitive MAC scheme Suzan Bayhan and Fatih Alagöz Boğaziçi University, Computer Engineering Department, İstanbul.

2. Varalakshmi, S., \& Shanmugavel, S. (2014). Adaptive mac layer spectrum sensing algorithms for cognitive radio networks, International Journal of Advanced Information Science and Technology (IJAIST), 30(30), 45-52.

3. Sengottuvelan, S., Ansari, J., Mähönen, P., Venkatesh, T. G., \& Petrova, M. (2017). Channel selection algorithm for cognitive radio networks with heavy-tailed idle times. IEEE Transactions on Mobile Computing, 16(5), 1258-1271.

4. Akyildiz, I. F., Lo, B. F., \& Balakrishnan, R. (2011). Cooperative spectrum sensing in cognitive radio networks: A survey. Physical Communication, 4, 40-62.

5. Sriharipriya, K. C., \& Baskaran, K. (2015). A survey on cooperative spectrum sensing techniques for cognitive radio networks. International Journal of Engineering and Advanced Research Technology (IJEART), 1(6), 17-22.

6. Lu, Y., Wang, D., \& Fattouche, M. (2016). Cooperative spectrum-sensing algorithm in cognitive radio by simultaneous sensing and BER measurements. EURASIP Journal on Wireless Communications and Networking, 2016, 136.

7. Arora, N., \& Mahajan, R. (2014). Cooperative spectrum sensing using hard decision fusion scheme. International Journal of Engineering Research and General Science, 2(4), 36-43.

8. Ganesan, G., \& Li, Y. (2007). Cooperative spectrum sensing in cognitive radio, part I: Two user networks. IEEE Transactions on Wireless Communications, 6, 2204-2213.

9. Kim, S., \& Giannakis, G. B. (2010). Sequential and cooperative sensing for multi-channel cognitive radios. IEEE Transactions on Signal Processing, 58(8), 4239-4253.

10. Goel, A., \& Singh, J. (2015). Decision oriented hybrid spectrum sensing approach in cognitive radio for OFDM systems. International Journal of Advancement in Engineering Technology, Management \& Applied Science 2(9).

11. Ejaz, W., ul Hasan, N., Azam, M. A., \& Kim, H. S. (2012). Improved local spectrum sensing for cognitive radio networks. EURASIP Journal on Advances in Signal Processing, 2012, 242.

12. Khalid, L. (2014). Efficient techniques for cooperative spectrum sensing in cognitive radio networks. Dissertation, Ryerson University.

13. Chen, Y., Zhao, Q., \& Swami, A. (2008). Joint design and separation principle for opportunistic spectrum access in the presence of sensing errors. IEEE Transactions on Information Theory, 54(5), 2053-2071.

14. Edward, C. Y. P., Ying-Chang, L., Yong, L. G., \& Yong Hong, Z. (2009). Optimization of cooperative sensing in cognitive radio networks: A sensing-throughput tradeoff view. IEEE Transactions on Vehicular Technology, 58(9), 5294-5299.

15. Zhang, W., Mallik, R. K., \& Letaief, K. B. (2009). Optimization of cooperative spectrum sensing with energy detection in cognitive radio networks. IEEE Transactions on Wireless Communications, 8(12), 5761-5766. 
16. Aghaei, F., \& Avokh, A. (2020). MRCSC: A cross-layer algorithm for joint multicast routing, channel selection, scheduling, and call admission control in multi-cell multi-channel multi-radio cognitive radio wireless networks. Pervasive and Mobile Computing, 64, 101150.

17. Zikria, Y. B., Ishmanov, F., Afzal, M. K., Kim, S. W., Nam, S. Y., \& Yu, H. (2018). Opportunistic channel selection MAC protocol for cognitive radio ad hoc sensor networks in the internet of things. Sustainable Computing: Informatics and Systems, 18, 112-120.

18. Slimeni, F., Chtourou, Z., Scheers, B., Le Nir, V., \& Attia, R. (2019). Cooperative Q-learning based channel selection for cognitive radio networks. Wireless Networks, 25(7), 4161-4171.

19. Nagul, S. (2020). Channel scheduling by spectrum channel white space filling in cognitive radio networks. In 2020 IEEE international conference on electronics, computing and communication technologies (CONECCT) (pp. 1-6) IEEE.

20. Velempini, M., \& Dlodlo, M. E. (2009). Analyzing the effects of increasing data channels and the number of data flows on network performance. In 2009 1st international conference on wireless communication, vehicular technology, Information Theory and Aerospace \& Electronic Systems Technology, Aalborg, Denmark (pp. 600-605). https://doi.org/10.1109/WIRELESSVITAE.2009.5172514.

21. Salameh, H. B., Shraideh, S., \& Alshamali, A. (2020). Joint channel assignment and adaptive mode selection in MIMO-based cognitive radio networks. Arabian Journal for Science and Engineering, 45(12), 10233-10244.

22. Maurya, D. K., Knit, S., \& Singh, R. K. (2020). Smart channel selection approach for cognitive radio networks. International Journal of Research and Development in Applied Science and Engineering (IJRDASE), 20(2), ISSN: 2454-6844.

23. Sumathi, D., \& Manivannan, S. S. (2020). Stochastic approach for channel selection in cognitive radio networks using optimization techniques. Telecommunication Systems, 76, 1-20.

24. Sumathi, D., \& Manivannan, S. S. (2020). Machine learning-based algorithm for channel selection utilizing preemptive resume priority in cognitive radio networks validated by ns-2. Circuits, Systems, and Signal Processing, 39(2), 1038-1058.

25. Balasubramanian, M., \& Rajamani, V. (2020). Energy harvesting in cognitive networks using feasible channel selection assignment. Recent Advances in Computer Science and Communications, 13(2), 173-182.

26. Qureshi, M. A., \& Tekin, C. (2020). Rate and channel adaptation in cognitive radio networks under time-varying constraints. IEEE Communications Letters, 24(12), 2979-2983.

27. Ghosh, C., Roy, S., \& Rao, M. B. (2012). Modeling and validation of channel idleness and spectrum availability for cognitive networks. IEEE Journal on Selected Areas in Communications, 30(10), 2029-2039.

28. Kim, H., \& Shin, K. G. (2006). Adaptive MAC-layer sensing of spectrum availability in cognitive radio networks. University of Michigan, Tech. Rep. CSE-TR-518-06.

Publisher's Note Springer Nature remains neutral with regard to jurisdictional claims in published maps and institutional affiliations.

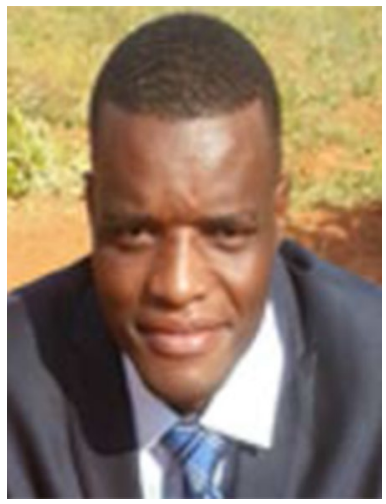

Joseph Tlouyamma is a Computer Science lecturer with research interest in Cognitive Radio Networks and 5G. 


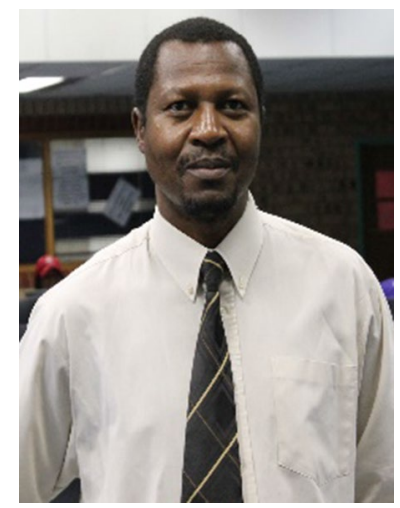

Mthulisi Velempini is an IEEE member and active researcher in Medium Access Control protocols, Routing protocols and Security in Computers. He is an emerging researcher in Wireless Access Network Technologies. 